\title{
Production of keV sterile neutrinos in supernovae: New constraints and gamma-ray observables
}

\author{
Carlos A. Argüelles, ${ }^{1,2 *}$ Vedran Brdar, ${ }^{3, \dagger}$ and Joachim Kopp ${ }^{3,+}$ \\ ${ }^{1}$ Massachusetts Institute of Technology, Cambridge, Massachusetts 02139, USA \\ ${ }^{2}$ Wisconsin IceCube Particle Astrophysics Center, Madison, Wisconsin 53703, USA \\ ${ }^{3}$ PRISMA Cluster of Excellence, 55099 Mainz, Germany and Mainz Institute for Theoretical Physics, \\ Johannes Gutenberg-Universität Mainz, 55099 Mainz, Germany
}

(Received 7 June 2016; revised manuscript received 26 July 2018; published 26 February 2019)

\begin{abstract}
We study the production of sterile neutrinos in supernovae, focusing in particular on the $\mathrm{keV}-\mathrm{MeV}$ mass range, which is the most interesting range if sterile neutrinos are to account for the dark matter in the Universe. Focusing on the simplest scenario in which sterile neutrinos mix only with muon or tau neutrinos, we argue that the production of keV-MeV sterile neutrinos can be strongly enhanced by a MikheyevSmirnov-Wolfenstein (MSW) resonance, so that a substantial flux is expected to emerge from a supernova, even if vacuum mixing angles between active and sterile neutrinos are tiny. Using energetics arguments, this yields limits on the sterile neutrino parameter space that decrease to mixing angles on the order of $\sin ^{2} 2 \theta \lesssim 10^{-14}$ and are up to an order of magnitude stronger than those from X-ray observations. Although supernova limits suffer from larger systematic uncertainties than X-ray limits, they apply also to scenarios in which sterile neutrinos are not abundantly produced in the early Universe. We also compute the flux of $\mathcal{O}(\mathrm{MeV})$ photons expected from the decay of sterile neutrinos produced in supernovae but find that it is beyond current observational reach even for a nearby supernova.
\end{abstract}

DOI: 10.1103/PhysRevD.99.043012

\section{INTRODUCTION}

One of the most auspicious candidate particles for the dark matter in the Universe is the sterile neutrino-an electrically neutral fermion with a mass on the order of $\mathrm{keV}-\mathrm{MeV}$ that couples to ordinary matter only through a tiny mass mixing with Standard Model (SM) neutrinos $[1,2]$. In the simplest sterile neutrino scenarios, it is assumed that the abundance of sterile neutrinos $\nu_{s}$ is zero at the end of inflation, and they are later produced through their mixing with SM (active) neutrinos $\nu_{a}[3,4]$ (see also [5-7]). Experimental constraints on the mass of keV sterile neutrinos and their mixing with SM neutrinos arise from the measured DM relic density [8,9], from Pauli blocking (the Tremaine-Gunn bound) [10,11], from Lyman- $\alpha$ forests [12-14] and from X-ray searches for radiative decays of sterile neutrinos $\nu_{s} \rightarrow \nu_{a}+\gamma$ [15-20]. From the combination of these constraints, one concludes that the $\nu_{s}$ mass should be $m_{s} \gtrsim 4 \mathrm{keV}$, and its mixing angle with the SM

\footnotetext{
caad@mit.edu

†vbrdar@uni-mainz.de

jkopp@uni-mainz.de
}

Published by the American Physical Society under the terms of the Creative Commons Attribution 4.0 International license. Further distribution of this work must maintain attribution to the author(s) and the published article's title, journal citation, and DOI. Funded by SCOAP ${ }^{3}$. neutrinos should be $\sin ^{2} 2 \theta \lesssim 10^{-6}$ in the simple two-flavor approximation.

In this paper, we add a new limit to this inventory of constraints by considering sterile neutrino production in core-collapse supernovae (SN) [21-31]. A supernova develops when a $\gtrsim 9 M_{\odot}$ star runs out of nuclear fuel. The thermal pressure that normally counteracts gravity disappears, and the core of the star collapses into a neutron star. The temperature in the nascent neutron star is $\gtrsim \mathrm{MeV}$, so that a thermal population of (active) neutrinos is produced. These $\nu_{a}$ can oscillate into $\nu_{s}$, which escapes the exploding star unhindered and may carry away significant amounts of energy [24,28,32]. Constraints on anomalous energy loss from SN 1987A will thus allow us to constrain the sterile neutrino parameters. As $\nu_{a} \rightarrow \nu_{s}$ conversion can be resonant thanks to the ultrahigh matter density $\sim 10^{14} \mathrm{~g} / \mathrm{cm}^{3}$ in the SN core, these limits will be very strong. The flux of sterile neutrinos with $\mathcal{O}(\mathrm{MeV})$ energies escaping from a supernova leads to a flux of secondary gamma-rays when they decay, and we study this flux, as well.

Our main results are summarized in Fig. 1. The solid orange exclusion region shows that, in the mass range $m_{s} \sim 2-80 \mathrm{keV}$, limits from energy loss in supernovae surpass previous limits by up to 2 orders of magnitude in $\sin ^{2} 2 \theta$ for the case when $\nu_{s}$ mixes with $\nu_{\mu}$ or $\nu_{\tau}$. Note that, unlike the other limits shown in Fig. 1, our bounds would still hold if sterile neutrinos are not part of the DM in the 


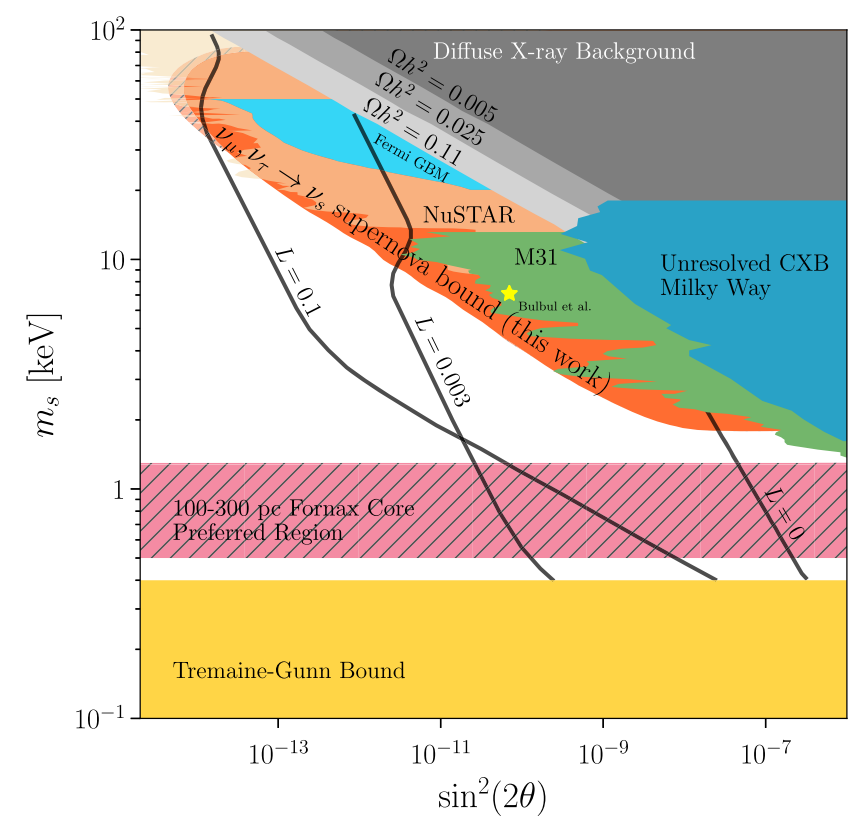

FIG. 1. Supernova bounds on the sterile neutrino mass $m_{s}$ and mixing $\sin ^{2} 2 \theta$ (orange, this work) between $\nu_{x}\left(x=\mu\right.$ or $\tau$ ) and $\nu_{s}$ flavors compared to previous constraints [17-19] from the Tremaine-Gunn bound [10,11] (yellow), NuSTAR observation of galactic center [33] (pale orange), X-ray searches in the Andromeda Galaxy M31 [15] (green), the Fermi GBM all-sky analysis [34] (purple), and the galactic [17] (dark blue) and extragalactic $[16,18,19]$ (gray) diffuse X-ray background. For the latter constraint, we show also how it is modified if sterile neutrinos account for only a fraction of the DM in the Universe. It is understood that the other X-ray limits would shift in a similar way if $\Omega h^{2}<0.11$. The hatched pink region indicates the region preferred by the properties of the core of the Fornax dwarf galaxy [35], whereas a yellow star shows the parameter point that could explain the $3.5 \mathrm{keV}$ X-ray line hinted at by Refs. [19,36]. Black curves illustrate the parameter regions in which the DodelsonWidrow mechanism [3] $(L=0)$ or the Shi-Fuller mechanism [4] with lepton assymetry $L>0$ would yield the correct DM relic density $\Omega h^{2}=0.11$. The hatched band at large $m_{s}$ indicates the theoretical uncertainty in our limit stemming from our treatment of energy transport inside the supernova (see Appendix A).

Universe. In the following, we discuss in detail how we have obtained our new limits and sensitivity estimates.

\section{STERILE NEUTRINO PRODUCTION IN SUPERNOVAE}

We consider a simplified two-flavor oscillation picture with mixing between a sterile neutrino ${ }^{1} \nu_{s}$ and one species of active neutrinos $\nu_{x}, x=\mu$ or $\tau$. We do not consider

\footnotetext{
${ }^{1}$ In this work, for brevity, the term "sterile neutrino" is denoting both sterile neutrinos and antineutrinos. The only exception is when discussing the adiabatic production where we refer explicitly to "sterile antineutrinos" because the resonance appears in the antineutrino channel.
}

mixing between $\nu_{s}$ and electron neutrinos to avoid complications arising from charged current interactions between $\nu_{e}$ and electrons/positrons. The flavor basis Hamiltonian describing neutrino propagation in matter includes the vacuum oscillation term and a MikheyevSmirnov-Wolfenstein (MSW) potential $V_{x}$ that describes coherent forward scattering of $\nu_{x}$ on the background matter via $Z$ exchange [37-39]:

$$
H=\frac{\Delta m^{2}}{4 E}\left(\begin{array}{cc}
-\cos 2 \theta & \sin 2 \theta \\
\sin 2 \theta & \cos 2 \theta
\end{array}\right)+\left(\begin{array}{cc}
V_{x} & 0 \\
0 & 0
\end{array}\right) .
$$

Here, $\theta \ll 1$ is the $\nu_{s}-\nu_{x}$ mixing angle in vacuum, and $\Delta m^{2} \simeq m_{s}^{2}$ is the mass squared difference between the two mass eigenstates. ${ }^{2}$ The MSW potential is $V_{x}=$ $\pm \sqrt{2} G_{F}\left(-N_{n} / 2+N_{\nu_{e}}-N_{\bar{\nu}_{e}}\right)$, where $G_{F}$ is the Fermi constant and $N_{n}, N_{\nu_{e}}$, and $N_{\bar{\nu}_{e}}$ are the neutron, electron neutrino, and electron antineutrino number densities, respectively. The $+(-)$ sign corresponds to the potential experienced by neutrinos (antineutrinos). As $\left|N_{\nu_{e}}-N_{\bar{\nu}_{e}}\right| \ll$ $N_{n} / 2$, the potential is positive for antineutrinos and negative for neutrinos. Note that we neglect terms that would arise from differences between the $\nu_{x}$ and $\bar{\nu}_{x}$ number densities because such differences are expected to be small in the parameter region where our limits will lie [32]. We have checked that even adding an MSW potential corresponding to a maximal asymmetry does not alter our results significantly. We also neglect the momentum and angular dependence of neutrino self-interactions $[40,41]$ and instead restrict ourselves to the simplified formalism used in $[24,25,28]$. Because $N_{n}, N_{\nu_{e}}$, and $N_{\bar{\nu}_{e}}$ are extremely large in the supernova core and gradually decrease with radius, most antineutrinos will encounter an MSW resonance on their way out of the exploding star. At the resonance,

$$
\cos 2 \theta=2 V_{x} E_{\mathrm{res}} / m_{s}^{2}
$$

and the effective mixing angle $\theta_{m}$ in matter becomes maximal [37-39,42].

We consider two physically different mechanisms for sterile neutrino production in supernovae:

(i) Adiabatic flavor conversion at an MSW resonance. A $\bar{\nu}_{x}$ of energy $E$ streaming away from the supernova core can convert to sterile antineutrino $\left(\bar{\nu}_{s}\right)$ when the matter density, and thus the MSW potential $V_{x}$, has reached the value satisfying the resonance condition, Eq. (2) [43]. Each point $(t, R)$ in time and space corresponds to a specific value of $V_{x}$; therefore, antineutrinos of a specific energy $E_{\text {res }}(t, R)$ are resonantly converted at this point. We take the local antineutrino luminosities and spectra and the local matter densities from the

\footnotetext{
${ }^{2}$ In this paper, we refer to $m_{s}$ as the sterile neutrino mass, even though we mean of course the mass of the heavy mass eigenstate which is mostly a $\nu_{s}$, with only a tiny admixture of $\nu_{x}$.
} 
simulation of an $8.8 M_{\odot}$ supernova by the Garching group [44] (see also [45-48]).

Hard scattering processes must be rare to give antineutrinos sufficient time to convert adiabatically. We therefore require the spatial width of the MSW resonance regions,

$$
R_{\text {width }}=2 \sin 2 \theta\left(\frac{1}{V_{x}} \frac{\partial V_{x}}{\partial R}\right)^{-1}
$$

to be smaller than the mean free path $\lambda_{\mathrm{mfp}}$. The number $d \mathcal{N}_{s}^{\mathrm{MSW}} / d E$ of $\bar{\nu}_{s}$ in an energy interval $[E, E+d E]$ produced by adiabatic flavor conversion at the MSW resonance is given by [24]

$$
\begin{aligned}
\frac{d \mathcal{N}_{s}^{\mathrm{MSW}}}{d E}= & \int_{0}^{t} d t^{\prime} 4 \pi R_{\mathrm{res}}^{2} n_{\nu}\left(t^{\prime}, R_{\mathrm{res}}\right) \\
& \times f_{x}(E) P_{\mathrm{res}}(E) \frac{E^{2}}{\bar{E}^{3}} \Theta\left(\lambda_{\mathrm{mfp}}-R_{\text {width }}\right)
\end{aligned}
$$

Here, the time integral runs from the time of core bounce $\left(t^{\prime}=0\right)$ until $\sim 9 \mathrm{~s}$ later, and $R_{\text {res }}\left(t^{\prime}, E\right)$ is the radius at which the resonance energy is $E$ at time $t^{\prime}$. The quantity $n_{\nu}\left(t^{\prime}, R_{\text {res }}\right)$ is the active (anti)neutrino number density at time $t^{\prime}$ and radius $R_{\text {res }}, f_{x}(E)$ is the energy distribution of active (anti)neutrinos, $\bar{E}$ is the average (anti)neutrino energy, $P_{\text {res }}(E)$ is the flavor conversion probability at the resonance, and the Heaviside $\Theta$ function implements the condition that antineutrinos must have enough time between collisions to convert adiabatically. It is crucial that antineutrinos do not encounter more than one MSW resonance on their way out of the supernova. (This would be different if we considered mixing between $\nu_{s}$ and $\nu_{e}$ instead of $\nu_{\mu, \tau}$ $[26,27]$.) Note that we can make the strongly simplifying assumption of radial symmetry, and we also neglect the depletion of active antineutrinos by conversion into $\bar{\nu}_{s}$. Moreover, we do not need to consider $\bar{\nu}_{x}$ streaming inwards. They would convert to $\bar{\nu}_{s}$ at the resonance, then travel through the core, and convert back to $\bar{\nu}_{x}$ on its far side.

We parameterize $f_{x}(E)$ as [49]

$f_{x}(E)=\frac{(1+\alpha)^{3+\alpha}}{\Gamma(3+\alpha)}\left(\frac{E}{\bar{E}}\right)^{\alpha} \exp \left[-(1+\alpha) \frac{E}{\bar{E}}\right]$

with normalization $\int_{0}^{\infty} d E E^{2} f_{x}(E)=\bar{E}^{3}$. (This relation defines $\bar{E}$.) The "pinching parameter" $\alpha$ desribes the degree to which $f_{x}(E)$ differs from a MaxwellBoltzmann distribution.

The $\bar{\nu}_{x} \rightarrow \bar{\nu}_{s}$ conversion probability at the resonance is given by the Landau-Zener formula $[24,25,42]$,

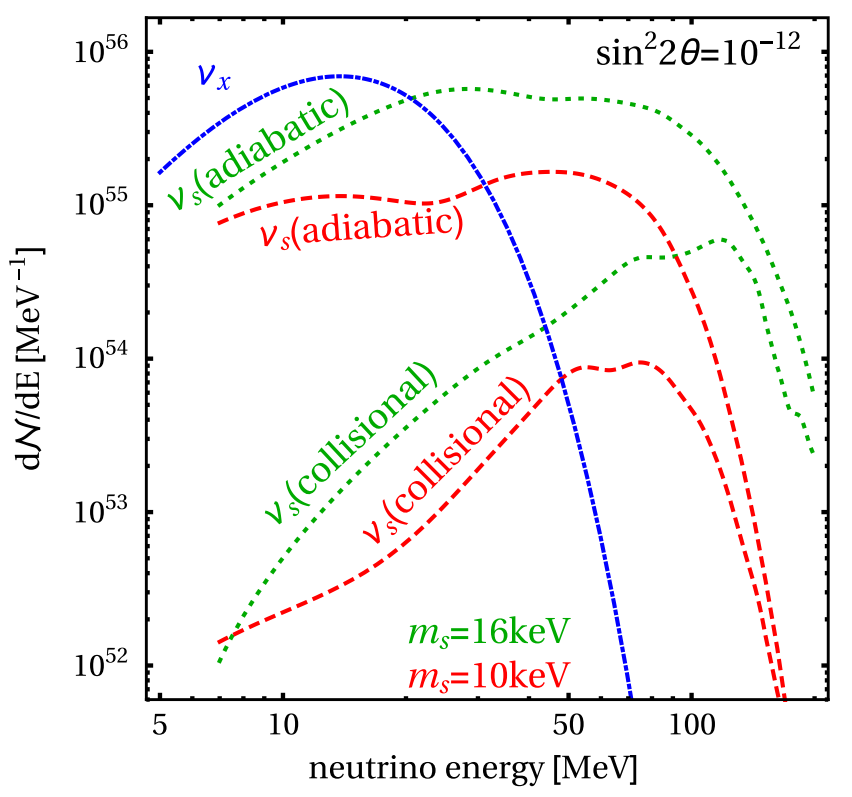

FIG. 2. Time-integrated spectra of active neutrinos (blue) and sterile neutrinos (green and red) produced in an $8.8 M_{\odot}$ supernova [44] during the first $\sim 9 \mathrm{~s}$ after the core bounce. Contributions from adiabatic flavor conversion and collisional production are shown separately. The $\nu_{s}$ spectrum extends to much higher energies than the $\nu_{x}$ spectrum because flavor conversion occurs in a region where temperatures are much higher than those at the last scattering surface of $\nu_{x}$.

$$
P_{\text {res }}(E)=1-\exp \left[-\frac{\pi^{2}}{2} \frac{R_{\text {width }}}{L_{\text {osc }}^{\text {res }}}\right]
$$

with the oscillation length at the resonance, $L_{\mathrm{osc}}^{\mathrm{res}} \simeq 2 \pi /\left(V_{x} \sin 2 \theta\right)$.

We find that adiabatic flavor conversion occurs mostly at radii $\sim 10-15 \mathrm{~km}$, still inside the neutrino sphere at $\sim 20-30 \mathrm{~km}$. In Fig. 2, we compare $d \mathcal{N}_{s}^{\mathrm{MSW}} / d E$ to the spectrum of active neutrinos. We see that the sterile antineutrino spectrum extends to higher energies because most of the flavor conversion happens in a high-temperature region from which $\nu_{s}$ can stream out freely, while $\nu_{x}$ are still trapped.

(ii) Collisional production. Sterile neutrinos can also be produced from a mixed $\nu_{x}-\nu_{s}$ state in hard scatterings on nucleons, electrons, and positrons. We take the interaction rate $\Gamma_{x}$ to be approximately equal to the dominant scattering rate on neutrons, with the corresponding cross section given by $[50,51]$ $\sigma_{\nu n} \simeq G_{F}^{2} E_{\nu}^{2}\left(1+g_{A}^{2}\right) / 4 \pi$, with $g_{A} \simeq 1.23$. The physical picture for collisional $\nu_{s}$ production is as follows: starting with an ensemble of only $\nu_{x}$ at $t=0$, each of them soon acquires a small $\nu_{s}$ admixture $\propto \sin ^{2} 2 \theta_{\mathrm{m}}$ by oscillation. A collision causes the collapse of the resulting mixed state into either a pure $\nu_{x}$ or a pure $\nu_{s}$. Afterwards, oscillations start anew, and $\nu_{x}$ are 
quickly replenished. After many collisions, the $\nu_{s}$ abundance is proportional to $\sin ^{2} 2 \theta_{\mathrm{m}}$ and $\Gamma_{x}$.

Up to a factor of $1 / 2$, this intuitive picture leads to the correct quantum mechanical Boltzmann equation $[1,3,23,52,53]$,

$$
\frac{\partial}{\partial t} \frac{d n_{s}}{d E}=\frac{\Gamma_{x}}{2}\left\langle P_{\nu_{x} \rightarrow \nu_{s}}\right\rangle \frac{d n_{x}}{d E} .
$$

Here, $d n_{s} / d E$ and $d n_{x} / d E$ are the energy spectra of sterile and active neutrinos, respectively. At any given spacetime point, $d n_{x}(t, R, E) / d E$ is related to the distribution function in Eq. (5) via $\left(1 / n_{x}\right) d n_{x} / d E=\left(E^{2} / \bar{E}^{3}\right) f_{x}(E)$. The averaged oscillation probability $\left\langle P_{\nu_{x} \rightarrow \nu_{s}}\right\rangle$ is [1]

$$
\left\langle P_{\nu_{x} \rightarrow \nu_{s}}\right\rangle=\frac{1}{2} \frac{\sin ^{2} 2 \theta}{\left(\cos 2 \theta-2 V_{x} E / m_{s}^{2}\right)^{2}+\sin ^{2} 2 \theta+\left(\Gamma_{x} E / m_{s}^{2}\right)^{2}} .
$$

The extra term $\left(\Gamma_{x} E / m_{s}^{2}\right)^{2}$ in the denominator compared to the usual expression for the mixing angle in matter [42] accounts for the suppression of $\nu_{s}$ production when $\lambda_{\mathrm{mfp}}$ is much smaller than the oscillation length, so that oscillations do not have time to develop between collisions (quantum Zeno effect).

Integrating Eq. (7) over time and radius leads to the energy spectrum of sterile neutrinos produced collisionally,

$$
\frac{d \mathcal{N}_{s}^{\text {coll }}}{d E}=\int_{0}^{t} d t^{\prime} \int_{0}^{R_{\text {core }}} d R^{\prime} 4 \pi R^{\prime 2} \frac{\Gamma_{x}}{2}\left\langle P_{\nu_{x} \rightarrow \nu_{s}}\right\rangle \frac{d n_{x}}{d E} .
$$

We again evaluate $d \mathcal{N}_{s} / d E$ numerically using the data from [44]. The resulting $\nu_{s}$ spectra, shown in Fig. 2, can be harder than the ones from adiabatic production because the collisional production rate depends on $\Gamma_{x}$, which grows proportional to $E^{2}$.

\section{CONSTRAINTS FROM SUPERNOVA LUMINOSITY}

We can constrain the energy output in sterile neutrinos from SN 1987A by comparing the observed energy output in active neutrinos of $\mathcal{E}_{a}=$ few $\times 10^{53} \mathrm{ergs}[54,55]$ to the gravitational energy released in the collapse of a stellar core at the Chandrasekhar mass, which is also on the order of $\mathcal{E}_{\text {tot }}=$ few $\times 10^{53}$ ergs $[44,56]$. If a substantial fraction of $\mathcal{E}_{\text {tot }}$ was carried away by sterile neutrinos, the observed $\mathcal{E}_{a}$ could not be explained. ${ }^{3}$ We therefore consider the ratio $\mathcal{R}\left(\sin ^{2} 2 \theta, m_{s}\right) \equiv \mathcal{E}_{s}\left(\sin ^{2} 2 \theta, m_{s}\right) / \mathcal{E}_{\text {tot }} \quad$ [21]. We assume

\footnotetext{
${ }^{3}$ A similar constraint could also be obtained by considering the observed duration of the neutrino emission from SN 1987A. Efficient production of sterile neutrinos would expedite the cooling of the supernova core, shortening the neutrino burst $[28,32]$.
}

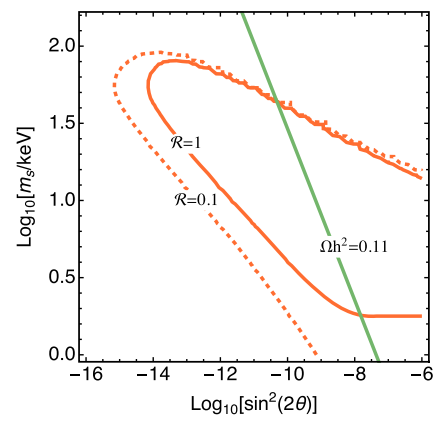

(a)

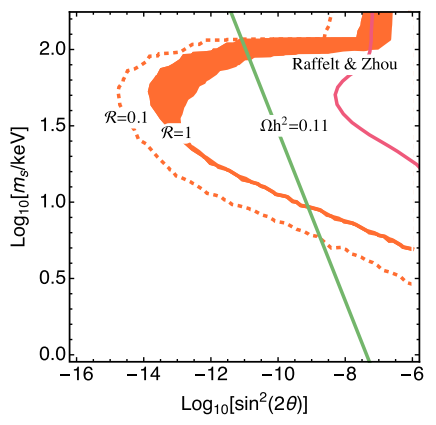

(b)
FIG. 3. Constraints on the sterile neutrino parameter space from energy loss in supernovae, considering (a) adiabatic $\nu_{x} \rightarrow \nu_{s}$ conversion only or (b) collisional $\nu_{s}$ production only. The solid and dotted orange curves correspond to different assumptions on the maximum allowed energy loss, expressed here in terms of the ratio $\mathcal{R}$ of the energy output in sterile neutrinos and the total energy output. For comparison, we also show in green the parameters for which the Dodelson-Widrow mechanism [3] predicts the correct relic abundance of $\nu_{s}$. In panel (b) for $\mathcal{R}=1$, the shaded region illustrates the theoretical uncertainty in our limit stemming from our treatment of energy transport inside the supernova (see Appendix A).

that $\mathcal{R}$ depends only weakly on the mass and type of the progenitor star, so that the values obtained for the supernova simulated in [44] are a good proxy for SN 1987A. Indeed, the energy emitted in neutrinos from the simulated supernova is roughly $1.5 \times 10^{53} \mathrm{erg}$ [44], which is on the same order of magnitude as in Supernova 1987A.

Our computation of $\nu_{s}$ production is only self-consistent for $\mathcal{R} \ll 1$ because we neglect depletion of active neutrinos. Extrapolating it to larger values nevertheless and setting a limit by requiring $\mathcal{R}<1$ yields the leftmost edge of the hatched uncertainty band in Fig. 1. To obtain the right edge of the band, we have also implemented a more robust treatment of active neutrino fluxes that parametrizes both depletion due to $\nu_{s}$ production and replenishment from diffusion (see Appendix A for details).

Our limits on the $\nu_{s}$ parameter space are shown in Fig. 3 for adiabatically and collisionally produced $\nu_{s}$ separately and in Fig. 1 for the combination of both production mechanisms (requiring $\mathcal{R}<1$ ). Due to MSW enhancement, our constraints become $\sin ^{2} 2 \theta \sim 10^{-14}$ at $m_{s} \sim 10-100 \mathrm{keV}$, surpassing all other limits in this mass range. The strength of our bounds can be understood by noting that, according to Eq. (8), strong resonant conversion is possible down to $\sin ^{2} 2 \theta \sim 10^{-14}$. At smaller values, the damping term $\left(\Gamma_{x} E / m_{s}^{2}\right)^{2}$ in the denominator suppresses $\left\langle P_{\nu_{x} \rightarrow \nu_{s}}\right\rangle$ at resonance. Moreover, considering the smooth density profiles from [44] rather than assuming a constant density core as, e.g., in Ref. [28] implies that, in the mass range between $10-100 \mathrm{keV}$, neutrinos of any energy will experience a resonance somewhere along their trajectory. It is primarily due to this effect that the limits on $\sin ^{2} 2 \theta$ presented here are 
(in the most sensitive region around $m_{s}=50 \mathrm{keV}$ ) about 3-4 orders of magnitude stronger than those in Ref. [28].

The shape of the exclusion regions in Fig. 3 can be understood as follows. For adiabatic conversion at small $m_{s}$, the oscillation length at the MSW resonance $L_{\text {osc }}^{\text {res }}$ is large, making flavor conversion nonadiabatic according to Eq. (6). At large $m_{s} \gtrsim 100 \mathrm{keV}$, the resonance condition of Eq. (2) cannot be satistifed. At $m_{s} \sim$ few $\times 10 \mathrm{keV}$, adiabatic flavor conversion is most effective at low $\sin ^{2} 2 \theta \sim 10^{-14}$. For larger $\sin ^{2} 2 \theta$, the radial width $R_{\text {width }}$ of the resonance region becomes too large, so that neutrinos scatter before having a chance to convert. Also, collisional production is most effective when the mixing angle in matter is MSW enhanced. At $m_{s} \lesssim 10 \mathrm{keV}$, the resonance condition of Eq. (2) is fulfilled only in the outer regions of the supernova, where the scattering rate is too low for effective collisional production. At $m_{s} \gtrsim 100 \mathrm{keV}$, the resonance condition is never satisfied.

\section{PHOTON FLUX FROM $\nu_{s} \rightarrow \nu_{a} \gamma$}

Sterile neutrinos, once produced, decay to photons via $\nu_{s} \rightarrow \nu_{a} \gamma$, a process mediated by loop diagrams involving charged leptons and $W$ bosons. The decay rate is $[57,58]$

$\Gamma_{\nu_{s} \rightarrow \nu_{a} \gamma}=1.38 \times 10^{-29} \sec ^{-1}\left(\frac{\sin ^{2} 2 \theta}{10^{-7}}\right)\left(\frac{m_{s}}{1 \mathrm{keV}}\right)^{5}$.

Thus, if $\nu_{s}$ are abundantly produced in a supernova, we expect the explosion to be accompanied by a flux of energetic $[\mathcal{O}(1-100 \mathrm{MeV})]$ secondary gamma-rays. Photons in this energy range are not normally expected from a supernova or supernova remnant, both of which emit $\mathrm{X}$-rays only at energies $\lesssim 10 \mathrm{keV}[59,60]$. The arrival times of the gamma-rays from $\nu_{s}$ decay are spread out over a time interval,

$$
\Delta t \simeq 3.6 \mathrm{hrs} \times\left(\frac{d}{1 \mathrm{kpc}}\right)\left(\frac{m_{s}}{1 \mathrm{keV}}\right)^{2}\left(\frac{1 \mathrm{MeV}}{E_{\gamma}}\right)^{2},
$$

where $d$ is the distance to the supernova and $E_{\gamma}$ is the gamma-ray energy. Sterile neutrinos decaying immediately after their production lead to gamma-rays that reach the Earth at the same time as the active neutrino burst. Gammarays from $\nu_{s}$ decaying only after traveling a distance $\simeq d$ are delayed by $\Delta t$.

The photon flux from $\nu_{s}$ decay in units of $\mathrm{cm}^{-2} \mathrm{~s}^{-1} \mathrm{MeV}^{-1}$ is given by

$\frac{d \phi_{\gamma}\left(E_{\gamma}\right)}{d E_{\gamma}}=\frac{2}{4 \pi \Delta t d^{2}}\left[1-\exp \left(-\frac{d}{\gamma} \Gamma_{\nu_{s} \rightarrow \nu_{a} \gamma}\right)\right] \frac{d \mathcal{N}_{\nu_{s}}\left(E_{\nu}\right)}{d E_{\nu}}$,

where $\gamma=2 E_{\gamma} / m_{s}$ is the Lorentz boost factor. The factor in brackets accounts for the $\nu_{s}$ decay probability. Note that the

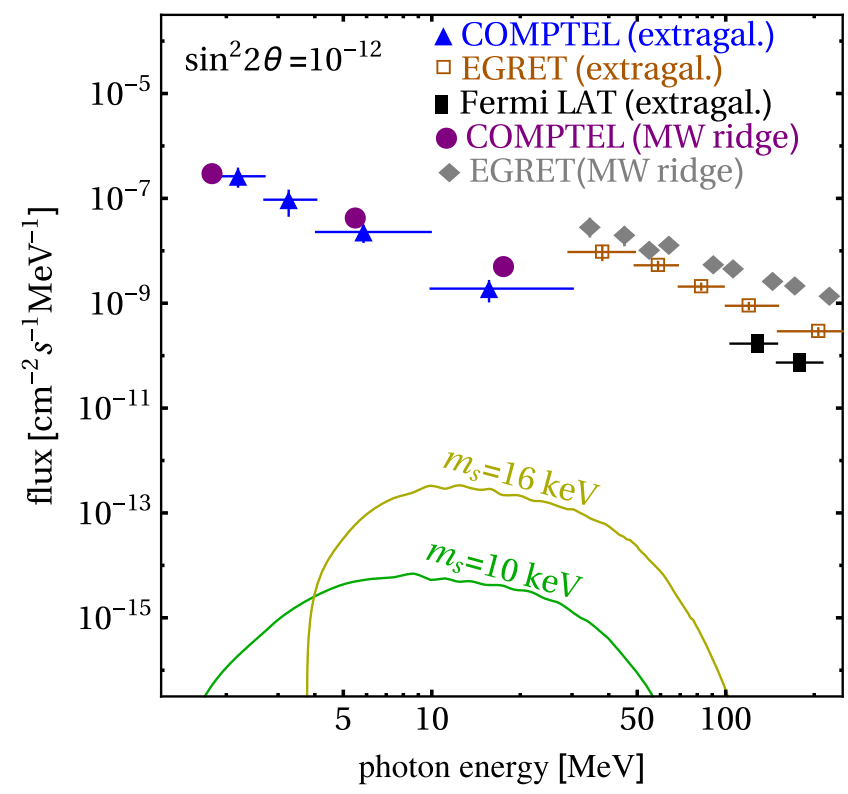

FIG. 4. Photon flux from the decay of sterile neutrinos produced in a nearby $(d=1 \mathrm{kpc})$ supernova for two different benchmark points in the $m_{s}-\sin ^{2} 2 \theta$ plane. We compare to observed spectra of diffuse gamma-rays from COMPTEL [61-63], EGRET [64-66], and Fermi-LAT [67], assuming angular bin sizes of 1 degree for COMPTEL, 5 degrees for EGRET, and 3 degrees for Fermi-LAT [71].

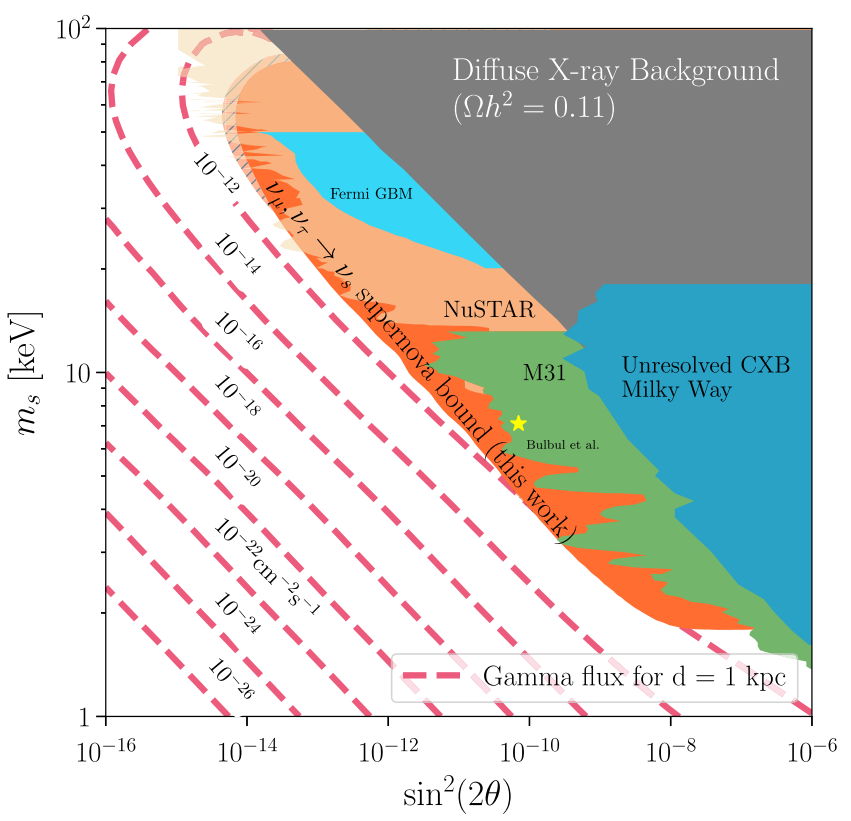

FIG. 5. Expected photon flux from the decay of sterile neutrinos produced in a supernova at distance $d=1 \mathrm{kpc}$. We compare to our new energy loss limits from Fig. 1 (orange region) and to constraints from X-ray searches [15-19,34] (green, dark blue, gray, and light blue regions). 
sterile neutrino spectrum $d N_{\nu_{s}}\left(E_{\nu}\right) / d E_{\nu}$ is evaluated at $E_{\nu}=2 E_{\gamma}$.

We plot $d \phi_{\gamma}\left(E_{\gamma}\right) / d E_{\gamma}$ for two sets of benchmark parameters in Fig. 4, and the total gamma-ray flux as a function of $\sin ^{2} 2 \theta$ and $m_{s}$ is shown in Fig. 5. As expected from Fig. 2, and taking into account $E_{\gamma}=E_{\nu} / 2$, the photon spectrum peaks at $E \sim 10-100 \mathrm{MeV}$. Compared to observations [61-67], we find that the signal is still several orders of magnitude below the uncertainty on the background. A direct observation of the $\nu_{s}$-induced photon signal is therefore challenging even for the next generation of Compton telescopes [68-70] and would require a factor $\sim 10^{5}$ improvement compared to the projected sensitivity of ComPair [69] for a supernova at $1 \mathrm{kpc}$.

\section{DISCUSSION AND CONCLUSIONS}

In conclusion, we have computed the flux of hypothetical $\mathrm{keV}-\mathrm{MeV}$ sterile neutrinos $\nu_{s}$ from a supernova. We have then used our results to constrain the $\nu_{s}$ parameter space using energy loss arguments (Fig. 1). Let us summarize once again the assumptions and approximations on which these limits are based: (i) we constrain mixing of $\nu_{s}$ with $\nu_{\mu}$ or $\nu_{\tau}$, but not with $\nu_{e}$; (ii) we rely on the supernova simulation from [44] being a good proxy for SN 1987A, but in terms of the total energy output and in terms of the time-dependent matter density and neutrino flux profiles; (iii) we use an approximate treatment of energy transport in the supernova, which is relevant for the replenishment of neutrinos that have converted into $\nu_{s}$ (Appendix A). Although we expect our results to be correct at the order-of-magnitude level, a more refined treatment, e.g., by implementing $\nu_{\mu, \tau} \rightarrow \nu_{s}$ conversion directly in a hydrodynamical supernova simulation, is certainly desirable. On the other hand, it is important to note that limits derived from supernovae are independent of the cosmological abundance of sterile neutrinos; i.e., they apply even to scenarios where $\nu_{s}$ production in the early Universe is suppressed or where $\nu_{s}$ are diluted after their production.

In the last part of the paper, we have estimated the gammaray flux from $\nu_{s}$ produced in a nearby supernova. However, we conclude that observing such a signal will be challenging even for the next generation of Compton telescopes. The signal may come within reach if sterile neutrinos exist with the parameters suggested by the $3.5 \mathrm{keV} \mathrm{X}$-ray line found in Refs. $[19,36]$ and if a very nearby supernova $(\lesssim 100$ pc) occurs.

Directions for future work include a more detailed calculation of the $\nu_{s}$ flux from a supernova, going beyond the two-flavor approximation and with a more detailed treatment of collective effects.

\section{ACKNOWLEDGMENTS}

We are indebted to Georg Raffelt and Ninetta Saviano for many useful comments on the manuscript, and to Thomas Janka and his collaborators for providing the data from [44] in machine-readable form. We would also like to thank Markus Ackermann, John Beacom, Alexei Smirnov, MacKenzie Warren, and Shun Zhou for very useful discussions. V. B. would like to thank the Wisconsin IceCube Particle Astrophysics Center (WIPAC) for hospitality. The work of V.B. and J.K. is supported by the German Research Foundation (DFG) under Grants No. KO 4820/ 1-1 and No. FOR 2239 and by the European Research Council (ERC) under the European Union's Horizon 2020 research and innovation programme (Grant Agreement No. 637506, " $\nu$ Directions"). V. B. is supported by the DFG Graduate School Symmetry Breaking in Fundamental Interactions (GRK 1581). Additional support has been provided by the Cluster of Excellence "Precision Physics, Fundamental Interactions and Structure of Matter" (PRISMA-EXC 1098), Grant No. 05H12UME of the German Federal Ministry for Education and Research (BMBF). C. A. was supported in part by the National Science Foundation (OPP-0236449, NSF-PHY1505858, and PHY-0969061) and by the University of Wisconsin Research Committee with funds granted by the Wisconsin Alumni Research Foundation.

\section{APPENDIX A: DEPLETION OF ACTIVE NEUTRINOS}

In our treatment of adiabatic and collisional production of sterile neutrinos, we did not yet address in detail the impact of active neutrino depletion on the $\nu_{s}$ production rate. More precisely, when active neutrinos are converted to $\nu_{s}$ in the MSW resonance region, their number density in this region quickly decreases. Simultaneously, neutrinos from elsewhere in the supernova diffuse into the resonance region, partially compensating the loss due to $\nu_{s}$ production. In the following, we present our method for taking depletion and diffusion into account.

The differential equations governing active and sterile neutrino energy transport to and from a small volume element $V$ in a time window $t$ are

$$
\begin{gathered}
\frac{1}{V} \frac{d E_{a}}{d t}=-\Gamma_{a s}+\Gamma_{s a}+I_{a}-O_{a}, \\
\frac{1}{V} \frac{d E_{s}}{d t}=\Gamma_{a s}-\Gamma_{s a}+I_{s}-O_{s} .
\end{gathered}
$$

Here, $E_{a}\left(E_{s}\right)$ is the total energy carried by the active (sterile) neutrinos in the volume element $V$ at radius $r, \Gamma_{a s}$ $\left(\Gamma_{s a}\right)$ is the rate of energy transfer by $\nu_{a} \rightarrow \nu_{s}\left(\nu_{s} \rightarrow \nu_{a}\right)$ conversion, and $I_{s(a)}$ and $O_{s(a)}$ represent the inflow and outflow of energy in sterile (active) neutrinos. All of these quantities should be understood to be functions of radius $r$ and time $t$. We will not include $I_{s(a)}$ and $O_{s(a)}$ directly but will implement a simplified treatment of neutrino transport at the very end of the following derivation. From Eq. (9), $\Gamma_{a s}$ reads 


$$
\Gamma_{a s}=\int d E \frac{\Gamma_{x}}{2}\left\langle P_{\nu_{x} \rightarrow \nu_{s}}\right\rangle n_{x} \frac{E^{2}}{\bar{E}^{3}} f_{x}(E) E .
$$

$\Gamma_{s a}$ is obtained by replacing the active neutrino energy distribution and number density with the corresponding quantities for sterile neutrinos. This term is, however, negligible because the strongest $\nu_{s}$ production occurs at the MSW resonance, and we can assume that they encounter no more than one resonance [see discussion below Eq. (4)]. Motivated by the relation $n_{x} \bar{E}=E_{a} / V$, we also define

$$
\tilde{\Gamma}_{a s} \equiv \frac{1}{V} \int d E \frac{\Gamma_{x}}{2}\left\langle P_{\nu_{x} \rightarrow \nu_{s}}\right\rangle \frac{E^{2}}{\bar{E}^{3}} f_{x}(E) E .
$$

With this definition, and with the simplification mentioned above, Eqs. (A1) and (A2) turn into

$$
\begin{gathered}
\frac{1}{V} \frac{d E_{a}}{d t}=-\frac{E_{a}}{\bar{E}} \tilde{\Gamma}_{a s}, \\
\frac{1}{V} \frac{d E_{s}}{d t}=\frac{E_{a}}{\bar{E}} \tilde{\Gamma}_{a s} .
\end{gathered}
$$

By solving Eq. (A5) for $E_{a}$ and inserting the solution into Eq. (A6), we arrive at

$$
\frac{1}{V} \frac{d E_{s}}{d t}=\frac{E_{a}(r, 0)}{\bar{E}} \tilde{\Gamma}_{a s} \exp \left[-\frac{1}{V} \int_{t, \Delta r} d V^{\prime} d t^{\prime} \frac{\tilde{\Gamma}_{a s}}{\bar{E}}\right],
$$

where $E_{a}(r, 0)$ is energy stored in active neutrinos at radius $r$ at the beginning of the supernovae cooling phase.

We now model the inflow and outflow terms by convoluting Eq. (A7) with a Gaussian whose width $\sigma$ represents the scale for active neutrino transport during the cooling phase:

$$
\begin{aligned}
\frac{1}{V} \frac{d E_{s}}{d t}= & \tilde{\Gamma}_{a s} \int_{V} d \hat{V} \frac{E_{a}(r, 0)}{\bar{E}} G(r, \mu=\hat{r}, \sigma=\sigma) \\
& \times \exp \left[-\frac{1}{\hat{V}} \int_{t, \Delta r=\hat{V}} d V^{\prime} d t^{\prime} \frac{\tilde{\Gamma}_{a s}}{\bar{E}}\right],
\end{aligned}
$$

where the Gaussian is a truncated Gaussian in the $\left[0, r_{\mathrm{SN}}\right]$ interval given by

$$
G(r, \mu, \sigma)=\kappa(\mu, \sigma) e^{\frac{(r-\mu)^{2}}{2 \sigma^{2}}} \theta(r) \theta\left(r_{\mathrm{SN}}-r\right),
$$

with $\kappa(\mu, \sigma)$ being the appropriate normalization factor to integrate to unity and $\theta$ the Heaviside function. We have also implemented an alternative phenomenological procedure in which the exponential that describes depletion of active neutrinos in Eq. (A7) is modified. In particular, we include an extra factor that suppresses the impact of depletion over time:
$\frac{1}{V} \frac{d E_{s}}{d t}=\frac{E_{a}(r, 0)}{\bar{E}} \tilde{\Gamma}_{a s} \exp \left[-\frac{1}{V} \int_{t, \Delta r} d V^{\prime} d t^{\prime} \frac{\tilde{\Gamma}_{a s}}{\bar{E}} \frac{t-t^{\prime}}{\sigma}\right]$.

In other words, the depletion factor at time $t$ depends mostly on $\nu_{s}$ production that happened recently, whereas the $\nu_{a}$ that have been converted to $\nu_{s}$ longer ago $\left(t-t^{\prime} \gtrsim \sigma\right)$ are assumed to have been replenished by diffusion.

We have verified that we obtain similar results using both techniques. The one based on Eq. (A10) is more stable and produces fewer numerical artifacts. The results obtained by applying the phenomenological based procedure, using $\tau$, are presented in Fig. 3.
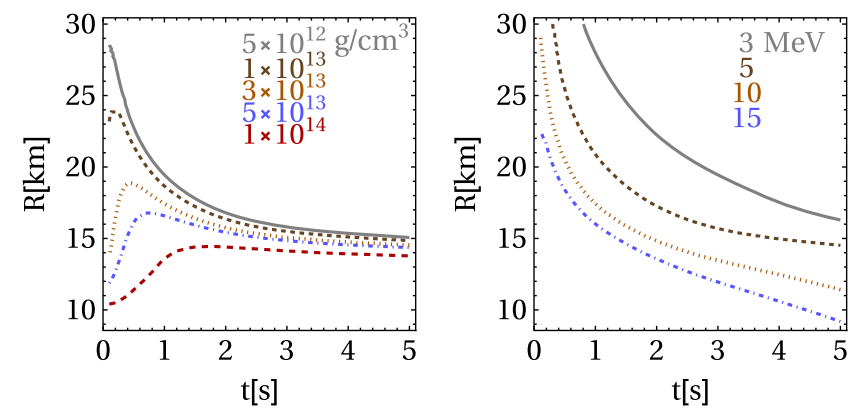

FIG. 6. (Left) Supernova density as a function of time $t$ and radius $r$. (Right) Supernova temperature as a function of $t$ and $r$. The leftmost edge of the plots at $t=0$ corresponds to the onset of the cooling phase.

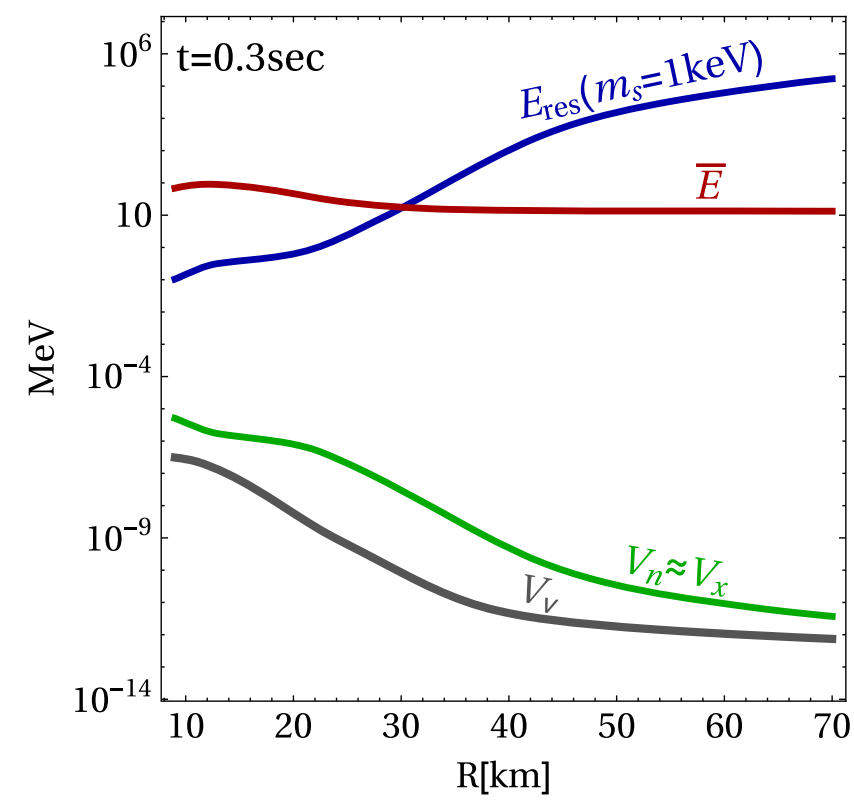

FIG. 7. For a specific point in time, $t=0.3 \mathrm{~s}$, we plot the MSW resonance energy $E_{\text {res }}$ (blue), the average neutrino energy $\bar{E}$ (red), and the MSW potential generated by ordinary matter $V_{n}$ (green) and by neutrinos $V_{\nu}$ (gray) as a function of radius $r$. 


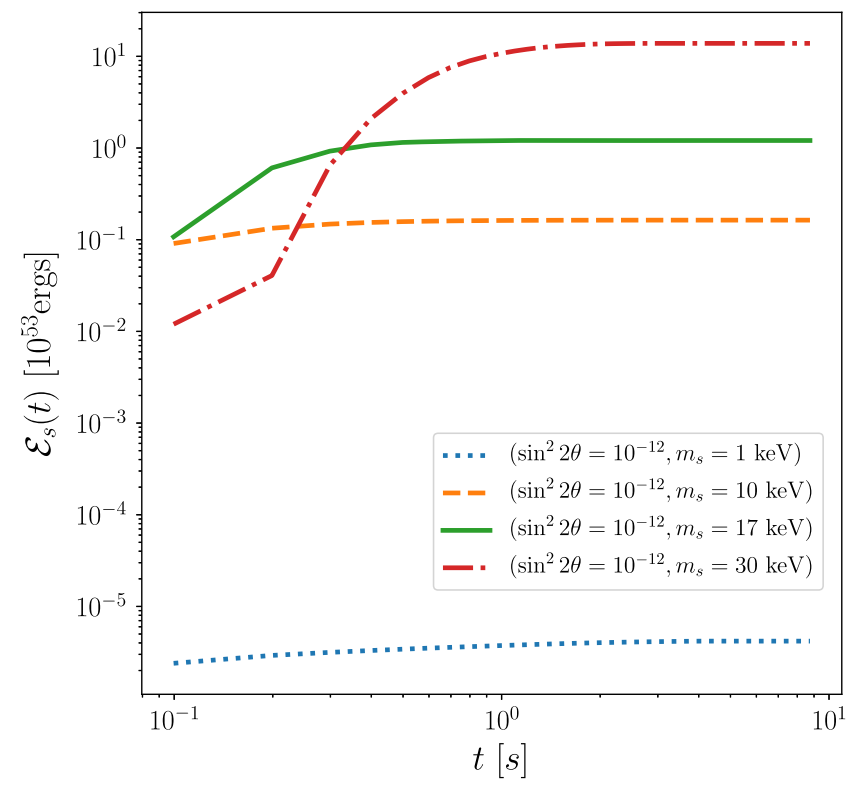

FIG. 8. Cumulative energy carried out of the supernovae explosion by collisionally produced sterile neutrinos for several parameter points.

\section{APPENDIX B: HYDRODYNAMICAL EVOLUTION AND ENERGY BUDGET}

In this Appendix, we show several ancillary plots illustrating the input and output of our simulations of sterile neutrino production in supernovae. In Fig. 6, we plot the properties of the supernova simulation from Ref. [44] on which the results of this paper are based. In particular, the two panels show contours of constant density (left) and temperature (right) as a function of the distance from the center of the star and of the times elapsed since the supernova bounce. We see that nuclear densities are reached in the inner core, and the supernova temperature is on the order of a few MeV.

Several quantities relevant to neutrino oscillations are plotted in Fig. 7 for a specific point in time at $t=0.3 \mathrm{~s}$ after the bounce. In particular, we compare the energy of the MSW resonance (blue) to the average neutrino energy (red).

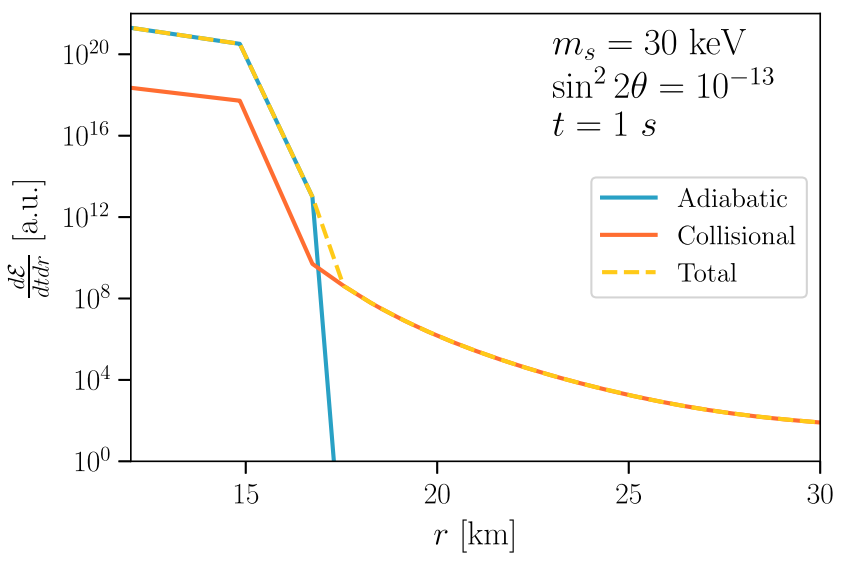

FIG. 9. Energy loss rate into $\nu_{s}$ via both collisional and adiabatic conversion for the parameter point $\sin ^{2} 2 \theta=10^{-13}$ and $m_{s}=30 \mathrm{keV}$ at $t=1 \mathrm{~s}$. Adiabatic contribution dominates at $r \lesssim 15 \mathrm{~km}$, where the resonance condition can be satisfied.

The intersection of the two curves indicates that the region favorable for adiabatic $\nu_{x} \rightarrow \nu_{s}$ conversion lies at a few tens of kilometers from the center of the star. We also show the contributions of coherent forward scattering on neutrinos $V_{\nu}$ (gray) and neutrons $V_{n}$ (green) to the total matter potential $V_{x}$.

In Fig. 8, we show the total integrated energy $\mathcal{E}_{e}$ carried out of the star by sterile neutrinos. We see that such transport is most efficient during the first second after the bounce. Comparing the different parameter points shown in Fig. 8, we observe that significantly more energy is lost into sterile neutrinos for $m_{s}>10 \mathrm{keV}$ than for masses below this threshold. The reason is that, at masses greater than $10 \mathrm{keV}$, resonant flavor conversion in matter is effective.

Finally, in Fig. 9, we plot the energy loss rate as a function of radius for a specific parameter point $\left(\sin ^{2} 2 \theta=10^{-13}\right.$, $m_{s}=30 \mathrm{keV}$ ). We see that both adiabatic and collisional conversion are relevant in the inner core $(r \lesssim 15 \mathrm{~km})$, with the adiabatic contribution being stronger. At larger radii, adiabatic conversion becomes ineffective because, due to the lower density, resonance energy increases to values outside the neutrino spectrum.
[1] K. Abazajian, G. M. Fuller, and M. Patel, Sterile neutrino hot, warm, and cold dark matter, Phys. Rev. D 64, 023501 (2001).

[2] A. Kusenko, Sterile neutrinos: The dark side of the light fermions, Phys. Rep. 481, 1 (2009).

[3] S. Dodelson and L. M. Widrow, Sterile-Neutrinos as Dark Matter, Phys. Rev. Lett. 72, 17 (1994).

[4] X.-D. Shi and G. M. Fuller, A New Dark Matter Candidate: Nonthermal Sterile Neutrinos, Phys. Rev. Lett. 82, 2832 (1999).
[5] K. Petraki and A. Kusenko, Dark-matter sterile neutrinos in models with a gauge singlet in the Higgs sector, Phys. Rev. D 77, 065014 (2008).

[6] A. Merle, V. Niro, and D. Schmidt, New production mechanism for keV sterile neutrino dark matter by decays of frozenin scalars, J. Cosmol. Astropart. Phys. 03 (2014) 028.

[7] A. Merle, A. Schneider, and M. Totzauer, Dodelson-Widrow production of sterile neutrino dark matter with non-trivial initial abundance, J. Cosmol. Astropart. Phys. 04 (2016) 003. 
[8] P. A. R. Ade et al. (Planck Collaboration), Planck 2015 results. XIII. Cosmological parameters, Astron. Astrophys. 594, A13 (2016).

[9] A. C. Vincent, E. F. Martinez, P. Hernandez, M. Lattanzi, and O. Mena, Revisiting cosmological bounds on sterile neutrinos, J. Cosmol. Astropart. Phys. 04 (2015) 006.

[10] S. Tremaine and J.E. Gunn, Dynamical Role of Light Neutral Leptons in Cosmology, Phys. Rev. Lett. 42, 407 (1979).

[11] D. Iakubovskyi, Constraining properties of dark matter particles using astrophysical data, Ph.D. thesis, Leiden University, 2013.

[12] J. Baur, N. Palanque-Delabrouille, C. Yche, C. Magneville, and M. Viel, Lyman-alpha forests cool warm dark matter, in SDSS-IV collaboration meeting, 2015, J. Cosmol. Astropart. Phys. 08 (2016) 012.

[13] A. Merle and A. Schneider, Production of sterile neutrino dark matter and the $3.5 \mathrm{keV}$ line, Phys. Lett. B 749, 283 (2015).

[14] A. Schneider, Astrophysical constraints on resonantly produced sterile neutrino dark matter, J. Cosmol. Astropart. Phys. 04 (2016) 059.

[15] S. Horiuchi, P. J. Humphrey, J. Onorbe, K. N. Abazajian, M. Kaplinghat, and S. Garrison-Kimmel, Sterile neutrino dark matter bounds from galaxies of the Local Group, Phys. Rev. D 89, 025017 (2014).

[16] A. Boyarsky, A. Neronov, O. Ruchayskiy, and M. Shaposhnikov, Constraints on sterile neutrino as a dark matter candidate from the diffuse x-ray background, Mon. Not. R. Astron. Soc. 370, 213 (2006).

[17] K. N. Abazajian, M. Markevitch, S. M. Koushiappas, and R. C. Hickox, Limits on the radiative decay of sterile neutrino dark matter from the unresolved cosmic and soft X-ray backgrounds, Phys. Rev. D 75, 063511 (2007).

[18] K. N. Abazajian, Detection of dark matter decay in the X-ray, arXiv:0903.2040.

[19] E. Bulbul, M. Markevitch, A. Foster, R. K. Smith, M. Loewenstein, and S. W. Randall, Detection of an unidentified emission line in the stacked X-ray spectrum of galaxy clusters, Astrophys. J. 789, 13 (2014).

[20] N. Sekiya, N. Y. Yamasaki, and K. Mitsuda, A search for a $\mathrm{keV}$ signature of radiatively decaying dark matter with Suzaku XIS observations of the X-ray diffuse background, Publ. Astron. Soc. Jpn. 68, SP1 (2015).

[21] S. P. Mikheev and A. Yu. Smirnov, Resonant oscillations and limitations found on neutrino parameter values from the possible observation of a neutrino burst from the gravitational collapse of a star, Pis'ma Zh. Eksp. Teor. Fiz. 46, 11 (1987) [JETP Lett. 46, 10 (1987)].

[22] K. Kainulainen, J. Maalampi, and J. T. Peltoniemi, Inert neutrinos in supernovae, Nucl. Phys. B358, 435 (1991).

[23] G. Raffelt and G. Sigl, Neutrino flavor conversion in a supernova core, Astropart. Phys. 1, 165 (1993).

[24] X. Shi and G. Sigl, A Type II supernovae constraint on electron-neutrino-sterile-neutrino mixing, Phys. Lett. B 323, 360 (1994); Erratum, Phys. Lett. B 324, 516 (1994).

[25] H. Nunokawa, J. T. Peltoniemi, A. Rossi, and J. W. F. Valle, Supernova bounds on resonant active sterile neutrino conversions, Phys. Rev. D 56, 1704 (1997).

[26] J. Hidaka and G. M. Fuller, Dark matter sterile neutrinos in stellar collapse: Alteration of energy/lepton number transport and a mechanism for supernova explosion enhancement, Phys. Rev. D 74, 125015 (2006).

[27] J. Hidaka and G. M. Fuller, Sterile neutrino-enhanced supernova explosions, Phys. Rev. D 76, 083516 (2007).

[28] G. G. Raffelt and S. Zhou, Supernova bound on keV-mass sterile neutrinos reexamined, Phys. Rev. D 83, 093014 (2011).

[29] M.-R. Wu, T. Fischer, L. Huther, G. Martnez-Pinedo, and Y.-Z. Qian, Impact of active-sterile neutrino mixing on supernova explosion and nucleosynthesis, Phys. Rev. D 89, 061303 (2014).

[30] M. L. Warren, M. Meixner, G. Mathews, J. Hidaka, and T. Kajino, Sterile neutrino oscillations in core-collapse supernova simulations, arXiv:1405.6101.

[31] M. Warren, G. J. Mathews, M. Meixner, J. Hidaka, and T. Kajino, A review of the impact of sterile neutrino dark matter on core-collapse supernovae, Int. J. Mod. Phys. A 31, 1650137 (2016).

[32] G. G. Raffelt, Astrophysical methods to constrain axions and other novel particle phenomena, Phys. Rep. 198, 1 (1990).

[33] K. Perez, K. C. Y. Ng, J. F. Beacom, C. Hersh, S. Horiuchi, and R. Krivonos, Almost closing the MSM sterile neutrino dark matter window with NuSTAR, Phys. Rev. D 95, 123002 (2017).

[34] K. C. Y. Ng, S. Horiuchi, J. M. Gaskins, M. Smith, and R. Preece, Improved limits on sterile neutrino dark matter using full-sky fermi-GBM data, Phys. Rev. D 92, 043503 (2015).

[35] L. E. Strigari, J. S. Bullock, M. Kaplinghat, A. V. Kravtsov, O. Y. Gnedin, K. Abazajian, and A. A. Klypin, A large dark matter core in the fornax dwarf spheroidal galaxy, Astrophys. J. 652, 306 (2006).

[36] A. Boyarsky, O. Ruchayskiy, D. Iakubovskyi, and J. Franse, An Unidentified Line in X-Ray Spectra of the Andromeda Galaxy and Perseus Galaxy Cluster, Phys. Rev. Lett. 113, 251301 (2014).

[37] L. Wolfenstein, Neutrino Oscillations in Matter, Phys. Rev. D 17, 2369 (1978).

[38] S. P. Mikheev and A. Yu. Smirnov, Resonance amplification of oscillations in matter and spectroscopy of solar neutrinos, Yad. Fiz. 42, 1441 (1985) [Sov. J. Nucl. Phys. 42, 913 (1985)].

[39] S. P. Mikheev and A. Yu. Smirnov, Resonant amplification of neutrino oscillations in matter and solar neutrino spectroscopy, Nuovo Cimento C 9, 17 (1986).

[40] H. Duan, G. M. Fuller, and Y.-Z. Qian, Collective neutrino oscillations, Annu. Rev. Nucl. Part. Sci. 60, 569 (2010).

[41] B. Dasgupta and A. Dighe, Collective three-flavor oscillations of supernova neutrinos, Phys. Rev. D 77, 113002 (2008).

[42] E. K. Akhmedov, Neutrino physics, arXiv:hep-ph/0001264.

[43] S. P. Mikheev and A. Yu. Smirnov, Neutrino oscillations in a variable density medium and neutrino bursts due to the gravitational collapse of stars, Zh. Eksp. Teor. Fiz. 91, 7 (1986) [Sov. Phys. JETP 64, 4 (1986)].

[44] L. Hüdepohl, B. Müller, H. Janka, A. Marek, and G. G. Raffelt, Neutrino Signal of Electron-Capture Supernovae from Core Collapse to Cooling, Phys. Rev. Lett. 104, 251101 (2010); Erratum, Phys. Rev. Lett. 105, 249901(E) (2010), machine-readable data from http:/wwwmpa.mpa-garching .mpg.de/ccsnarchive/data/Huedepohl2010-data/index.html; 
we use results for the model with full neutrino interactions (Sf).

[45] M. Rampp and H. T. Janka, Radiation hydrodynamics with neutrinos: Variable Eddington factor method for core collapse supernova simulations, Astron. Astrophys. 396, 361 (2002).

[46] M. Liebendoerfer, M. Rampp, H. T. Janka, and A. Mezzacappa, Supernova simulations with Boltzmann neutrino transport: A Comparison of methods, Astrophys. J. 620, 840 (2005).

[47] A. Marek, H. Dimmelmeier, H. T. Janka, E. Muller, and R. Buras, Exploring the relativistic regime with Newtonian hydrodynamics: An Improved effective gravitational potential for supernova simulations, Astron. Astrophys. 445, 273 (2006).

[48] R. Buras, M. Rampp, H. T. Janka, and K. Kifonidis, Two-dimensional hydrodynamic core-collapse supernova simulations with spectral neutrino transport. 1. Numerical method and results for a 15 solar mass star, Astron. Astrophys. 447, 1049 (2006).

[49] M. T. Keil, G. G. Raffelt, and H.-T. Janka, Monte Carlo study of supernova neutrino spectra formation, Astrophys. J. 590, 971 (2003).

[50] C. Giunti and C. W. Kim, Fundamentals of Neutrino Physics and Astrophysics (Oxford University Press, Oxford, UK, 2007).

[51] J. Peltoniemi, The ultimate neutrino page, http://cupp.oulu .fi/neutrino/.

[52] L. Stodolsky, On the Treatment of neutrino oscillations in a thermal environment, Phys. Rev. D 36, 2273 (1987).

[53] M. J. Thomson, Damping of quantum coherence by elastic and inelastic processes, Phys. Rev. A 45, 2243 (1992).

[54] T. J. Loredo and D. Q. Lamb, Bayesian analysis of neutrinos observed from supernova SN-1987A, Phys. Rev. D 65, 063002 (2002).

[55] G. Pagliaroli, F. Vissani, M. L. Costantini, and A. Ianni, Improved analysis of SN1987A antineutrino events, Astropart. Phys. 31, 163 (2009).

[56] K. Zuber, Neutrino Physics, Series in High Energy Physics, Cosmology and Gravitation (Taylor \& Francis, London, 2011), 2nd ed.

[57] P. B. Pal and L. Wolfenstein, Radiative decays of massive neutrinos, Phys. Rev. D 25, 766 (1982).

[58] Z. Xing and S. Zhou, Neutrinos in Particle Physics, Astronomy and Cosmology, Advanced Topics in Science and Technology in China (Springer, Berlin, Heidelberg, 2011).
[59] A. M. Soderberg et al., An extremely luminous X-ray outburst marking the birth of a normal supernova, Nature (London) 453, 469 (2008).

[60] J. Vink, Supernova remnants: The X-ray perspective, Astron. Astrophys. Rev. 20, 49 (2012).

[61] A. W. Strong, K. Bennett, H. Bloemen, R. Diehl, W. Hermsen, D. Morris, V. Schoenfelder, J. G. Stacy, C. de Vries, M. Varendorff, C. Winkler, and G. Youssefi, Diffuse continuum gamma rays from the Galaxy observed by COMPTEL, Astron. Astrophys. 292, 82 (1994).

[62] S. C. Kappadath, J. Ryan, K. Bennett, H. Bloemen, D. Forrest, W. Hermsen, R. M. Kippen, M. McConnell, V. Schoenfelder, R. van Dijk, M. Varendorff, G. Weidenspointner, and C. Winkler, The preliminary cosmic diffuse $\gamma$-ray spectrum from $800 \mathrm{keV}$ to $30 \mathrm{MeV}$ measured with COMPTEL, Astron. Astrophys. Proc. Suppl. 120, 619 (1996).

[63] R. L. Kinzer, W. R. Purcell, and J. D. Kurfess, Gamma-ray emission from the inner galactic ridge, Astrophys. J. 515, 215 (1999).

[64] S. D. Hunter et al., EGRET observations of the diffuse gamma-ray emission from the galactic plane, Astrophys. J. 481, 205 (1997).

[65] P. Sreekumar et al. (EGRET Collaboration), EGRET observations of the extragalactic gamma-ray emission, Astrophys. J. 494, 523 (1998).

[66] P. Sreekumar, F. W. Stecker, and S. C. Kappadath, The extragalactic diffuse gamma-ray emission, AIP Conf. Proc. 410, 344 (1997).

[67] M. Ackermann et al. (Fermi-LAT Collaboration), The spectrum of isotropic diffuse gamma-ray emission between $100 \mathrm{MeV}$ and $820 \mathrm{GeV}$, Astrophys. J. 799, 86 (2015).

[68] S. D. Hunter et al., A pair production telescope for mediumenergy gamma-ray polarimetry, Astropart. Phys. 59, 18 (2014).

[69] A. A. Moiseev et al., Compton-pair production space telescope (ComPair) for $\mathrm{MeV}$ gamma-ray astronomy, arXiv:1508.07349.

[70] M. Tavani et al. (e-ASTROGAM Collaboration), Science with e-ASTROGAM (A space mission for $\mathrm{MeV}-\mathrm{GeV}$ gamma-ray astrophysics), arXiv:1711.01265.

[71] V. Schönfelder and G. Kanbach, Imaging through Compton scattering and pair creation, Observing Photons in Space: A Guide to Experimental Space Astronomy (Springer, New York, 2013), pp. 225-242. 